\title{
CIENCIA, IDEALIZACIÓN Y PREDICCIÓN. LOS TIPOS IDEALES EN MAX WEBER Y LOS CONCEPTOS IDEALES EN CIENCIAS DE LA NATURALEZA ${ }^{1}$
}

\section{SCIENCE, IDEALIZATION AND PREDICTION. MAX WE- BER'S IDEAL-TYPES AND IDEAL CONCEPTS IN NATURAL SCIENCES.}

\author{
Mariano Sanjuán ${ }^{2}$ \\ Universidad Autónoma de Madrid
}

\author{
Recibido: 17-02-2018 \\ Aceptado: 29-05-2018
}

\begin{abstract}
Resumen: El objetivo de este ensayo es exponer una comparativa acerca del carácter predictivo de los conceptos ideales en las ciencias de la naturaleza, por una parte, y su rol en los tipos-ideales weberianos, por otra. Se argumentará a favor de la capacidad predictiva de los primeros, y en contra de la de los segundos. Se presentará también un análisis conceptual del debate histórico $E r$ klären-Verstehen, al que pueden retrotraerse las tensiones vistas entre ambas propuestas. Por último, se expondrá una tipología de la noción de predicción que pueda aportar luz a una redefinición de la predicción en los tipos ideales.
\end{abstract}

Palabras clave: Idealización; Predicción; Weber; Ciencia; Metodología

\begin{abstract}
In this essay, I develop a comparative analysis of the role of prediction in ideal concepts within Natural Sciences, and its function in Weber's idealtypes. I will argue for the predictive capacity of the former and against of the latter. A conceptual analysis of the historical debate Erklären-Verstehen will be also presented. I will defend that our issue can adequately be traced back to that discussion. Finally, a typology of the notion of prediction will be exposed to open the way towards a redefinition of the predictive capacity of ideal-types.
\end{abstract}

Key-words: Idealization; Prediction; Weber; Science; Methodology

1. Agradezco los comentarios del profesor W. J. González y de dos revisores anónimos de la revista Thémata a versiones previas del presente artículo.

2. Profesor Ayudante de Lógica y Filosofía de la Ciencia de la Universidad Autónoma de Madrid Contacto: marianosanjuansalinas@gmail.com 


\section{Caracterización teórico-metodológica del concepto de idealización}

Las idealizaciones son un elemento nuclear en toda teoría científica, que no por ello está ausente de controversia. Su existencia ha recibido atención rigurosa en filosofía de la ciencia. Siguiendo a C. Liuª la noción de idealización refiere a teorizaciones que incluyen condiciones ideales físicamente plausibles, que no son el caso- y enunciados ideales -que son verdaderos syss el anterior elemento fuese el caso. Y según N. Cartwright ${ }^{4}$, en una idealización partimos de una realidad concreta a la que sometemos a los reajustes oportunos, de tal manera que se neutralicen las perturbaciones o inconvenientes que dificultan su conocimiento.

L. Nowak ${ }^{5}$, por su parte, ha distinguido cinco paradigmas distintos del concepto de idealización. Estos difieren, fundamentalmente, en el estatus de las idealizaciones, que van desde enunciados deliberadamente falsos a modelos aproximadamente fieles al mundo real. Con todo, estos enfoques mantienen un denominador común: toda conceptualización contiene cierto grado de idealización. En otras palabras: una idealización es un constituyente interno a la metodología de toda teoría científica y un recurso instrumental mediador entre la realidad de índole natural y/o social y nuestras operaciones sobre la misma.

La presencia de idealizaciones en la ciencia atiende esencialmente a dos objetivos: primero, proporcionar una explicación que integre los fenómenos estudiados en un sistema coherente y fundamentado; y segundo, abrir la puerta a juicios de carácter predictivo sobre el devenir de la parcela de la realidad considerada. Este trabajo pretende comparar dos sentidos concretos del concepto de idealización: los conceptos ideales en ciencias de la naturaleza-CI, de aquí en adelante-y los tipos-ideales en la propuesta metodológica de Max Weber -TI, de aquí en adelante ${ }^{6}$.

Las posiciones al respecto han argüido fundamentalmente en torno a dos cuestiones. La primera se relaciona con la capacidad de instanciación

3. Liu, C.: "Approximation, Idealization, and Laws of Nature" en Synthese 118, 1999, pp. 229-256.

4. Cartwright, N.: Nature's Capacities and their Measurement. Oxford: Oxford, 1989, especialmente pp. 183 y ss.

5. Nowak, L.: "The idealizational approach to science: a new survey" en Brezinski, J. y Nowak, L. (eds.). Idealization III: Approximation and Truth. Poznan Studies in the Philosophy of the Sciences and the Humanities. Ámsterdam/Atlanta: Rodopi, 1992, pp. 9-63.

6. Esta relación ha sido ya objeto de diversas reflexiones. Algunas de las más relevantes han sido: Hempel, C.G.: La explicación científica. Estudios sobre la Filosofía de la ciencia. Buenos Aires: Paidós, 1965/1978); Papineau, D.: "Ideal Types and Empirical Theories" en The British Journal for the Philosophy of Science 27 no. 2, 1976, pp. 137-146; Ramsey, J. L.: "Ideal Reaction Types and the Reactions of Real Alloys" en Proceedings of the Biennial Meeting of

Thémata. Revista de Filosofía №58 (2018) pp.: 13-34. 
de los TI, es decir, de encontrar manifestaciones reales de las entidades que recaen bajo los conceptos-límite que construyen. De ser posible, como afirman C. Hempel o Jeffry L. Ramsey, la segunda cuestión es un corolario de la primera: se trata de la asimilabilidad de los TI a los CI. Autores como Ibarra y Mormann ${ }^{7}$ o G. Camardi ${ }^{8}$ son partidarios de esta posibilidad. Esta perspectiva, paradójicamente, se antoja contraria a las intenciones de Max Weber, defensor del dualismo metodológico. De Donato, por su parte, admite esta posición con mayor recelo, especificando dos sentidos del concepto de TI, tratando de lidiar con el problema de la contrastación empírica de los CI -la cual defiende- y asumiendo, por otra parte, que estos no son instanciables.

Esta doble concepción de los tipos ideales fue anteriormente establecida por J. W. N. Watkins, quien distingue entre los "TI holísticos" y los "TI individualísticos". De acuerdo con esta concepción, "los tipos ideales holísticos pretendían brindar una perspectiva a vista de pájaro de los rasgos generales de una situación social completa, mientras que los tipos ideales individualísticos son construidos inspeccionando la situación de individuales reales, y abstrayendo a partir de ellos"9. En mi caracterización considero que ambos sentidos no son mutuamente excluyentes y, por tanto, ignoro deliberadamente la distinción; a lo sumo, es asumible aceptar que cada uno de ellos describe ciertas propiedades de los TI sin contradecir las del opuesto.

Los objetivos de la perspectiva aquí propuesta son diferentes a los de trabajos anteriores en un doble sentido. Primero, porque considero que el debate filosófico entre los CI y los TI es un correlato de una fuente de disidencia mayor que, históricamente, se ha conocido como la problemática metodológica Erklären-Verstehen, entre la explicación que proporcionan las ciencias de la naturaleza y la comprensión aportada por las tradicionalmente denominadas "ciencias del espíritu". Y segundo, porque mi foco de atención se establece sobre un asunto que considero fundamental para una intelección completa del estudio comparativo: el carácter predictivo de uno y otro tipo de idealización. Mi objetivo será indicar que una tipología

the Philosophy of Science Association 1, 1994, pp. 149-159; De Donato, X.: "El carácter de los tipos ideales weberianos y su relación con las ciencias naturales" en Diánoia 52 no. 59, 2007, pp. 151-177.

7. Ibarra, A. y Mormann, T.: "Datos, fenómenos y constructos teóricos: un enfoque representacional" en Theoria 13 no. 1, 1998, pp. 61-87.

8. Camardi, G.: "Ideal Types and Scientific Theories" en F. Coniglione et. al. (eds.). Idealization XI: Historical Studies on Abstraction and Idealization. Ámsterdam: Rodopi, 2004, pp. 273-285.

9. Watkins, J. W. N.: "Ideal Types and Historical Explanation" en The British Journal for the Philosophy of Science 3 no. 9, 1952, p. 24.

Thémata. Revista de Filosofía ํ58 (2018) pp.: 13-34. 
adecuada del concepto de predicción puede vincularse satisfactoriamente con la noción de TI.

\section{Los conceptos ideales en ciencias de la naturaleza}

Una caracterización ampliamente compartida en filosofía de la ciencia divide en tres los tipos de conceptos manejados en ciencia básica desde una perspectiva axiomática: clasificatorios, comparativos y métri$\cos ^{10}$. La idea de CI, sin embargo, no se ajusta apropiadamente a ninguno de ellos. Es preferible, a mi juicio, entender que los CI podrían constituir una cuarta categoría.

Los CI que se manejan en este ensayo son del siguiente tipo: péndulo simple, cuerpo rígido, gas ideal, vacío perfecto, etc. Todos ellos se caracterizan por mantener una serie de concesiones heurísticas por las que determinados aspectos de la parcela de la realidad que el concepto tiene por extensión permanecen estables. Por ejemplo: el concepto de "cuerpo rígido" establece que las posiciones relativas de las partículas de un sistema físico determinado no varían ante la influencia de fuerzas externas. Este enunciado describe un estado de las cosas irrealizable per se en el mundo natural. No es posible encontrar en la naturaleza un objeto cuyas partículas satisfagan completamente dicha propiedad ${ }^{11}$.

El concepto simplifica las condiciones reales que sí se dan en el mundo natural, permitiendo elaborar cálculos al otorgar valores matemáticos a los elementos descritos en el CI. Ernest Nagel comparte esta intuición cuando afirma que los CI establecen "casos límite" de los términos teóricos que lo conforman ${ }^{12}$. No encontraremos tal cosa como un cuerpo rígido natural, pero su conceptualización ayuda al desarrollo de la cinemática en mecánica clásica. Por tanto, los CI describen situaciones modélicas, en las

10. La distinción fue propuesta por Stegmüller, W.: Teoría y experiencia. Barcelona: Ariel, 1970/1979.

11. Desde el primer momento asumo que los CI son, en un sentido débil que será precisado, instanciables. Esta afirmación no es aproblemática, y, de hecho, en la medida en que estos conceptos deliberadamente idealizan ciertos aspectos de la realidad, algunos autores han defendido que se trata de generalizaciones no instanciables (Cfr. Papineau, D.: op. cit., pp. 137-139; De Donato: op. cit., p. 166). Por instanciación de un CI entiendo la correspondencia empírica aproximativa del concepto con los casos que tiene por extensión.

12. Cfr. Nagel, E.: "Assumptions in Economic Theory" en The American Economic Review 53, no. 2, 1963, pp. 214-216. Para Nagel, los términos teóricos -de los cuales están compuestos los CI- no tienen referencia observacional; son los enunciados observacionales los que, a través de las reglas de correspondencia, se encargan de dotarles de significado empírico. Aun así, Nagel reivindica la pertinencia de los CI. Su carácter idealizado - es decir, la ausencia de referencia- no condiciona su legitimidad siempre y cuando los CI vengan confirmados por los enunciados observacionales.

Thémata. Revista de Filosofía №58 (2018) pp.: 13-34. 
que se omiten ciertos determinantes considerados perturbaciones y/o se introducen condiciones -cláusulas ceteris paribus-con el fin de explicar y predecir el comportamiento de los fenómenos de la naturaleza.

Un concepto que ayuda a comprender el funcionamiento metodológico de los CI es la noción kuhniana de "ejemplar"13. Los ejemplares son problemas resueltos por una teoría que actúan como modelo del comportamiento de una determinada parcela de la realidad. Constituyen, por tanto, una explicación. Lo que me interesa, sin embargo, es cómo la asimilación de los CI condiciona la mirada del científico de manera análoga a cómo lo hacen los ejemplares. Para Kuhn, la resolución de problemas - y, por ende, la obtención de conocimiento - es un ejercicio de aprendizaje y aplicación de los ejemplares. A lo largo de su educación, los miembros de una comunidad científica adquieren la habilidad de establecer relaciones de semejanza entre uno o más ejemplares disponibles en la teoría y problemas empíricos que presenta la naturaleza. Una teoría comienza con un ejemplar y se expande a partir de él. Algo similar sucede en la aplicación de un CI. Por ejemplo, un individuo aprende durante su formación qué es un cuerpo rígido y trata de entrever en la naturaleza objetos y comportamientos similares. Esta habilidad de categorizar lo convierte en un taxonomista $^{14}$. A partir de ahí, la complejidad es una cuestión de grado; de aplicar el ejemplar en situaciones en las que la analogía no es evidente.

Pero un CI no solo participa en las explicaciones de una teoría: uno de los rasgos esenciales de los CI es su capacidad predictiva. Los CI estipulan un modo concreto de comportamiento de ciertas entidades, de manera que, de cumplirse determinadas variables y dados unos valores matemáticos determinados, seremos capaces de predecir situaciones futuras con un buen grado de precisión. A la vista de lo expuesto, la capacidad predictiva es una condición necesaria para la viabilidad del CI, pues es precisamente lo que garantiza su conexión con los fenómenos empíricos que caen bajo su dominio. De Donato, para justificar el estatus ontológico realista de los CI, establece la noción de aproximación:

lo interesante de los conceptos ideales es que estamos legitimados a aplicarlos a situaciones reales que satisfagan aproximadamente las condiciones involucradas en su caracterización. [...] Si, después de muchas observaciones y experimentos, ob-

13. Para una descripción detallada del concepto de ejemplar, véase Kuhn, T. S.: "Epílogo: 1969" en La estructura de las revoluciones científicas. Madrid: Fondo de Cultura Económica, 1962/2007, pp. 301-353 y Kuhn, T. S.: Segundos pensamientos sobre paradigmas. Madrid: Tecnos, 1974/1978, especialmente pp. 16-17.

14. En rigor, es la aplicación de un léxico -y no tanto de un ejemplar- lo que convierte al científico en un taxonomista, en el sentido de aplicar las categorías no trascendentales de una estructura léxica sobre los problemas que se estipulan como relevantes -es decir, los problemas no resueltos.

Thémata. Revista de Filosofía $\mathrm{N}^{\circ} 58$ (2018) pp.: 13-34. 
tenemos como resultado que de tales generalizaciones no se puede decir que sean ni siquiera aproximadamente satisfechas, podemos rechazarlas ${ }^{15}$.

La satisfacción de los CI en ciencia básica es aproximativa y se establece a partir de cálculos predictivos. Si, por ejemplo, la consideración de cuerpos rígidos nos permite establecer cálculos predictivos sobre la aceleración de ciertos objetos en mecánica clásica, parece que estamos legitimados a afirmar que el CI "cuerpo rígido" posee instancias aproximadas; o cuando menos, que su vínculo con la experiencia está asegurado pese a su carácter ideal. Por tanto, la capacidad predictiva de un CI es el elemento clave a la hora de establecer su grado de aproximación con el mundo. Tras numerosas predicciones satisfactorias, estamos en situación de afirmar que el CI es empíricamente adecuado.

\section{Los tipos ideales en la metodología de Max Weber}

La noción de TI es introducida por primera vez por Max Weber en 1904. La meta de su elucidación acerca del carácter de los TI es proporcionar a la metodología de las ciencias sociales un valor específico y genuino, distintivo del resto de disciplinas, sin renunciar a la pretensión de objetividad. Para Weber, las ciencias sociales son capaces de proporcionar un tipo de conocimiento objetivo, a partir de una metodología particular restringida al estudio de los denominados fenómenos sociales de los procesos de la cultura $^{16}$. El elemento central de dicha metodología no es otro que los TI. Respecto a ellos, Weber afirma lo siguiente:

Se los obtiene mediante el realce unilateral de uno o varios puntos de vista y la reunión de una multitud de fenómenos singulares [...] en un cuadro conceptual en sí unitario. Este, en su pureza conceptual, es inhallable empíricamente en la realidad: es una utopía que plantea a la labor historiográfica la tarea de comprobar, en cada caso singular, en qué medida la realidad se acerca o se aleja de ese cuadro ideal. [...] El tipo ideal tiene el significado de un concepto límite puramente ideal, respecto del cual la realidad es medida y comparada a fin de esclarecer determinados elementos significativos de su contenido empírico ${ }^{17}$.

Ante todo, Los TI son construcciones teóricas, abstracciones conceptuales de carácter funcional, cuyo propósito es servir de vara de medir de la realidad. Weber utiliza el concepto de "utopía" en un sentido etimológico: los TI son u-tópicos, porque no tienen instancias en el mundo real. La identidad de los TI no viene dada por establecer concesiones en forma de

15. De Donato: op. cit., p. 168.

16. Cfr. Weber, M.: "La objetividad cognoscitiva de la ciencia social y de la política social" en Ensayos sobre metodología sociológica. Buenos Aires: Amorrortu, 1904/2001, pp. 52 y ss.

17. Ibídem, pp. 79-82.

Thémata. Revista de Filosofía $\mathrm{N}^{\circ} 58$ (2018) pp.: 13-34. 
cláusulas ceteris paribus, ni de simplificar el comportamiento del mundo natural: en los TI no hay ningún elemento que se comprometa con el estado real de las entidades que describe. Son, más bien, un recurso obtenido por abstracción de procesos singulares que permite evaluar situaciones de la historia social. Constituyen, siguiendo a Watkins, una "representación a priori del mundo" 18 .

J. L. Ramsey aporta una distinción fundamental, tomada de $\mathrm{N}$. Cartwright ${ }^{19}$, cuando afirma que "los tipos ideales son abstracciones más que idealizaciones"20. J. Drysdale también se muestra partidario de dicha terminología ${ }^{21}$. Siguiendo esta conceptualización, una abstracción selecciona una serie de variables concretas y finitas de un fenómeno determinado y elabora su análisis a partir de ese conjunto restringido de propiedades, mientras que las idealizaciones no acotan un determinado número de variables, sino que las estudia todas a la vez -es decir, no son selectivas-, pero modifica el valor de una o varias de ellas. Esta idea está en sintonía con la de L. Nowak, quien señala que "grosso modo, una abstracción consiste en pasar de las propiedades $\mathrm{AB}$ a $\mathrm{A}$, mientras que una idealización consiste en pasar de AB a A-B"22.

En otras palabras, una abstracción recorta las características de un sistema sin manipularlas, mientras que una idealización recoge todas las propiedades existentes deformando al menos una de ellas. Dadas estas definiciones, lo conveniente parece afirmar que los CI son idealizaciones, mientras que los TI son abstracciones ${ }^{23}$. En este punto, estimo oportuno concretar un doble principio de no contradicción lógica, necesario para la

18. Watkins, J. W. N.: op. cit., p. 26. Watkins considera los TI entidades a priori, en la medida en que preceden al estudio de la realidad social, y no en función de su analiticidad. Los TI son expresiones sintéticas, a partir de las que se efectúa la investigación.

19. Cartwright, N.: op. cit., pp. 187 y ss.

20. Ramsey, J. L.: op. cit., p. 152.

21. Drysdale, J.: "How are Social-Scientific Concepts Formed? A Reconstruction of Max Weber's Theory of Concept Formation" en Sociological Theory 14 no. 1, 1996, pp. 71-88, especialmente pp. 81-82.

22. Nowak, L.: op. cit., p. 8.

23. Del hecho de que los TI constituyen abstracciones podemos deducir que de un mismo hecho social podemos extraer varios TI. Expresado de forma alternativa: para dar cuenta de un fenómeno dado, el científico social dispone de tantos TI como mundos posibles sea capaz de construir mentalmente. Esta idea ha sido destacada en Freund, J.: Sociología de Max Weber. Barcelona: Ediciones Península, 1966/1986, pp. 62 y ss. y más recientemente en Sánchez de Puerta, F. J.: "Los tipos ideales en la práctica: significado, construcciones, aplicaciones" en Empiria. Revista de Metodología de Ciencias Sociales 11, 2006, p. 18. 
constitución de un concepto típico ideal por abstracción desde los fenómenos sociales e históricos particulares.

En primer lugar, el TI debe ser coherente respecto al fenómeno estudiado: el TI "socialismo", construido expresamente para la comprensión del fenómeno "La Revolución Rusa de 1917", no puede subsumir expresiones del estilo "La Revolución Rusa sucedió en el periodo medieval", o "Napoleón derrocó al último zar". Esta primera cláusula garantiza el vínculo empírico con el mundo. En segundo lugar, el TI deber ser coherente respecto al conjunto de conceptos típico ideales que participan en la investigación: si el TI "socialismo" describe ciertas relaciones económicas, políticas y de poder desde una perspectiva determinada, el dominio de aplicación del TI "imperialismo" no puede ser coincidente. Esta doble condición es probablemente el único elemento restrictivo en la formulación de los TI.

Por lo que respecta a su funcionamiento, en cuanto constructos mentales, los TI son contrafácticos. Un TI describe un mundo posible en el que se reconstruyen los sucesos reales dadas unas causas determinadas. El estudio de caso de mayor renombre de Max Weber responde a esta descripción ${ }^{24}$. Para comprender el exponencial desarrollo económico de ciertos países del norte de Europa durante los siglos XVI y XVII, Weber estipula una serie de TI como "espíritu del capitalismo" o "ascetismo calvinista", creando un mundo posible en que la conexión causal de estos y otros TI da cuenta de dicho desarrollo.

A partir de la comparación contrafáctica de la imagen causal construida con los sucesos históricos, el científico social está en posición de comprender el fenómeno referido. Es por ello que esta metodología recibe el nombre de "sociología comprensiva" 25 . Desde esta perspectiva, la metodología típico ideal es efectiva para comprender sucesos acaecidos en el pasado. Ahora bien: los TI no pueden emitir juicios justificados sobre los sucesos futuros. Un TI es un instrumento cuya función es comprender un fenómeno histórico, no explicar el comportamiento posible de conjuntos de fenómenos relacionados con el TI. En otras palabras: los TI, al contrario que los CI, no tienen capacidad predictiva.

Se pueden aportar al menos tres razones al respecto. La primera de ellas ha sido indicada por Papineau ${ }^{26}$. Su argumentación parte de la

24. Cfr. Weber, M.: La ética protestante y el espíritu del capitalismo. Barcelona: Península, 1905/1969.

25. Weber, M.: "Sobre algunas categorías de la sociología comprensiva" Ensayos sobre metodología sociológica. Buenos Aires: Amorrortu, 1913/2001, p. 175.

26. Papineau, D.: op. cit., pp. 137-138.

Thémata. Revista de Filosofía №58 (2018) pp.: 13-34. 
asunción de que un TI no tiene instancias ${ }^{27}$ para evaluar, a continuación, dos situaciones posibles: aquella en que el TI aparece en el antecedente de una generalización, y otra en que se sitúa en el consecuente. Siguiendo los principios básicos de la lógica, cuando el TI se sitúa en el antecedente, la inferencia, sea esta una predicción, está empíricamente injustificada. No es factible derivar un estado futuro de las cosas de un constructo estrictamente mental. Por otra parte, aquellas generalizaciones que contienen un TI en su consecuente predicen situaciones futuras literalmente irrealizables.

La segunda de las razones es metodológica y tiene que ver con la clase de fenómenos que aspiran a explicar cada uno. Viene señalada por el propio Weber en el siguiente fragmento:

la significación de la configuración de un fenómeno cultural, y su fundamento, no pueden ser obtenidos, fundados y vueltos inteligibles a partir de un sistema de conceptos legales (leyes), por perfecto que fuere. [...] Lo significativo no coincide, naturalmente, en cuanto tal, con ninguna ley, y por cierto, tanto menos cuanto más general sea aquella ${ }^{28}$.

Para Weber, las ciencias de la naturaleza buscan subsumir en generalizaciones legaliformes conjuntos de situaciones -eventos-tipo, según De Donato ${ }^{29}$ - de características similares, en la forma de una predicción. El modelo de caída de graves de Galileo, por ejemplo, establece que para todo cuerpo en caída en condiciones de perfecto vacío, independientemente de su masa, su aceleración será constante. Esta ley, que utiliza uno o varios CI, predice el comportamiento esperable de un conjunto de situaciones similares.

Por contrapartida, la metodología weberiana en ciencias sociales sigue una dirección inversa. No se buscan enunciados generales de gran amplitud, como el modelo galileano, sino que cada fenómeno particular es comprendido a partir del uso de uno o varios $\mathrm{TI}^{30}$. Weber no estaba interesado en encontrar leyes explicativas del surgimiento de toda revolución, pero sí en estudiar las causas concretas de cada revolución particular. Este hecho supone un impedimento de cara a la predicción: si cada fenómeno

27. La noción que maneja Papineau es la de "ideal type concept", que subsume las dos que se manejan en este artículo (CI y TI). Estoy de acuerdo en que los TI, como construcciones mentales, no tienen instancias. Su extensión es el conjunto vacío. No obstante, y como se ha mencionado en un pasaje anterior, considero que los CI sí las tienen, pues su extensión no coincide con el conjunto vacío, sino con un conjunto de sistemas y/o entidades del mundo real.

28. Weber, M.: "La objetividad" cit., pp. 65-66.

29. De Donato: op. cit., p. 155.

30. Esta contraposición ha sido también mostrada por Nowak. Cfr. Nowak, L.: The structure of idealization. Towards a systematic interpretation of the marxian idea of science. Dordrecht: Springer, 1980, pp. 42 y ss.

Thémata. Revista de Filosofía ํ58 (2018) pp.: 13-34. 
histórico se estudia en su individualidad, las causas que lo administran no pueden extrapolarse de manera justificada a casos futuros.

El tercer y último argumento está íntimamente ligado al anterior. Puede introducirse como la especificidad del objeto de estudio de las ciencias sociales o la inescrutabilidad de los fenómenos humanos ${ }^{31}$. Este asunto merece especial atención, y su estudio se engloba en el amplio debate Erklären-Verstehen.

\section{Contexto histórico de la propuesta de Max Weber en el marco de la controversia Erklären-Verstehen}

La polémica entre los CI y los TI es comprensible a la luz de la disputa metodológica, originada a mediados del XIX y desarrollada a lo largo del XX, acerca del estatus de las ciencias humanas y sociales. El asentamiento y desarrollo de disciplinas como la sociología, la economía o la historia planteaban urgentes dilemas sobre la posibilidad de obtención de conocimiento objetivo y legítimo. Se debatía si estas nuevas ciencias debían ser acogidas bajo el manto del método científico o si, en su lugar, les correspondía una caracterización metodológica específica no equiparable a aquella de las ciencias tradicionales o de la naturaleza. Los primeros han sido recogidos en el bando de Erklären, mientras que los últimos son localizados en el extremo Verstehen. La cuestión de fondo es una propuesta monista de la actividad científica frente a una dualista.

Antes de continuar, considero pertinente remarcar que Erklären y Verstehen constituyen dos perspectivas que contraponen el significado de las nociones de explicación y comprensión; es decir, distinguen entre dos actividades cognoscitivas epistémicamente diferentes. Tal distinción fue proporcionada por los defensores de Verstehen, que rechazaban el reduccionismo metodológico del que sí eran partidarios los defensores de $E r$ klären.

Desde esta dicotomía, el método de las ciencias de la naturaleza proporciona explicaciones acerca de su objeto de estudio, mientras que el de las ciencias sociales proporciona un conocimiento comprensivo. La diferencia fundamental entre ambas concepciones estriba en que el concepto de explicación nos remite a una descripción cuantitativa -expresable en términos numéricos-, nomológica -subsumible a leyes naturales- y atemporal -independiente del contexto histórico- del comportamiento del fenómeno estudiado. Consecuentemente, el estatus epistémico de una explicación aspira a ser objetivo, reproducible y universal. En contraposición, el

31. Este asunto, y sobre todo, su importancia en la teoría de la formación de conceptos en Max Weber, es atendido con reposo en Drysdale, J.: op. cit., especialmente pp. 75-79.

Thémata. Revista de Filosofía №58 (2018) pp.: 13-34. 
concepto de comprensión se refiere a una descripción cualitativa, no nomológica y singular. Y, en consecuencia, el estatus epistémico de una descripción comprensiva es relativo, particular e históricamente determinado.

Esto perfila una primera exposición del concepto de comprensión como contrapartida del de explicación: se trata, ante todo, de un método condicionado por la alteridad absoluta del tipo de fenómeno que pretende caracterizar: los hechos sociales. El problema que concernirá a Weber será el de si, a pesar de la particularidad e individualidad de cada hecho social, es viable la construcción de una metodología comprensiva y objetiva a partes iguales. Para llegar a ello, es precisa una breve presentación del origen de la disputa Erklären-Verstehen.

Tres precedentes a nuestro autor son suficientes para poner al lector en situación: A. Comte, J. G. Droysen y W. Dilthey ${ }^{32}$. Comte es considerado el padre de la tradición positivista y con seguridad la figura que mejor encarna la visión monista en metodología de la ciencia. Para Comte, el espíritu positivo ${ }^{33}$ consiste en encontrar los principios lógicos que rigen el ordenamiento de la realidad en conjunto. A este espíritu se subordinan todas las disciplinas: desde la matemática básica hasta la compleja física social. Según la filosofía positiva, explicar un fenómeno social es sinónimo de encontrar las leyes a las que puede reducirse. Esta máxima resume las pretensiones más radicales de Erklären: un método, un objetivo, un tipo de explicación ${ }^{34}$. Años después, surge en Alemania un movimiento de resistencia al establecimiento del positivismo.

En 1858, con la publicación de la obra de Droysen Grundiss der Historik aparecen por primera vez los conceptos de explicación y comprensión como responsables del tipo de conocimiento obtenido en ciencias de la naturaleza e historia, respectivamente. Con Droysen surge el dualismo

32. Estas tres caracterizaciones bajo ningún concepto agotan el abanico que desglosan las numerosas posiciones esgrimidas históricamente. Una intelección íntegra de la cuestión debería considerar obras como Der Sinnhafte Aufbau der Sozialen Welt, de A. Schütz, Explanation and Understanding, de G. H. von Wright, Wahrheit und Methode, de H. G. Gadamer, o Theory of Communicative Action, de J. Habermas, entre muchas otras. Hágase patente que mi objetivo se limita a presentar los polos excéntricos del marco Erklären-Verstehen para situar la caracterización weberiana en tal esquema.

33. La exposición del concepto de espíritu positivo se encuentra en Comte, A.: Discurso sobre el espíritu positivo. Buenos Aires: Aguilar, 1844/1982, pp. 52 y ss.

34. A decir verdad, Comte nunca llegó a cuestionarse la dualidad metodológica que Dilthey si planteó. En este sentido, situar a Comte en el marco de este debate es poner a alguien que, en rigor, no formó parte. Con todo, su propuesta metodológica encaja de manera evidente con las pretensiones del polo Erklären, que más adelante, recogerían los positivistas del Círculo de Viena. 
metodológico. En su concepción, las distintas disciplinas son asimétricas en cuanto a sus concepciones epistemológica y metodológica.

Fue Dilthey, sin embargo, quien reflejó el dualismo para confrontar el carácter comprensivo de las ciencias sociales al de corte efecto-causal de las ciencias de la naturaleza. Los principales argumentos a favor de esta distinción fueron dos. El primero tiene que ver con el control que el ser humano tiene sobre sus propias acciones ${ }^{35}$, en tanto sujeto capaz de modificar a placer el curso de los acontecimientos esperables. Esto indudablemente plantea serios conflictos a la hora de establecer predicciones y/o explicaciones sobre su comportamiento. El segundo se postula a nivel epistemológico. Trata sobre la estructura del conocimiento social. A juicio de Dilthey, "las ciencias del espíritu no constituyen un todo con una estructura lógica que sería análoga a la articulación que nos ofrece el conocimiento natural; su conexión se ha desarrollado de otra manera y es menester considerar cómo ha crecido históricamente" 36 .

El elemento histórico, sostiene Dilthey, es epistemológicamente significativo a la hora de comprender los fenómenos humanos. Ciertamente, les provee de una particularidad ausente en los objetos de estudio de las ciencias de la naturaleza. Por ello, su análisis desde una perspectiva histórica adquiere una primacía crucial, y sobretodo, diferenciada de las ciencias empíricas. Dilthey concluye que la irreconciabilidad es de tal magnitud que las ciencias del espíritu no coinciden con los valores de verdad y objetividad de las ciencias de la naturaleza, y deben consecuentemente emprender su propio camino. Si la propuesta de Comte suponía un extremo de la dicotomía Erklären-Verstehen en el ala Erklären, la de Dilthey se sitúa en el polo simétrico del lado Verstehen.

Se hace patente que las divergencias metodológicas tienen su origen en el tipo de realidad estudiada. Si las ciencias de la naturaleza eran capaces de explicar el comportamiento de su objeto de estudio, era debido a que se consideraba estable, y consecuentemente, el conocimiento obtenido a partir de un método correcto era totalmente objetivo. Por contrapartida, las ciencias sociales no eran capaces de aclarar con la misma precisión el comportamiento de su objeto de estudio y debían contentarse con comprenderlo, porque su objeto de estudio se consideraba mutable, y consecuentemente, el conocimiento obtenido es, a lo sumo, parcialmente objetivo. Ante tal dilema, los positivistas -Comte, primero, y más tarde el Círculo de Viena- trataron de objetivizar las ciencias sociales poniéndolas bajo la ad-

35. Cfr. Dilthey, W.: Introducción a las ciencias del espíritu, en las que se trata de fundamentar el estudio de la sociedad y de la historia. México: Fondo de cultura económica, 1883/1949, pp. 13 y ss.

36. Ibídem, pp. 31-32.

Thémata. Revista de Filosofía №58 (2018) pp.: 13-34. 
ministración del método de las ciencias de la naturaleza, al tiempo que los antipositivistas reivindicaron la comprensión y el conocimiento subjetivo como el camino a seguir por los científicos sociales.

La propuesta weberiana supone una reformulación genuina de los planteamientos así entendidos ${ }^{37}$. Weber acepta la demarcación metodológica entre unas ciencias y otras, pero no las deficiencias de objetividad achacadas a las ciencias sociales. Es posible, a su juicio, establecer una metodología viable, cuyo elemento clave son los TI, que sea comprensiva y objetiva a partes iguales. Según nuestro autor, la objetividad de las ciencias sociales no debe entenderse bajo el marco de la neutralidad valorativa ni de la reductibilidad a leyes normativas, de la manera en que se entienden y se reducen los fenómenos empíricos ${ }^{38}$.

La idea de objetividad weberiana está conectada con el hecho de que el objeto de estudio de las ciencias sociales sea susceptible de evaluación epistémica. Esto no está presente en las consideraciones más extremas de Verstehen. Su postura defiende la comprensión de los fenómenos sociales a partir de los TI, manteniendo así una distancia considerable con las perspectivas monistas. Pero a su vez, los TI constituyen explicaciones objetivas de un hecho histórico o social determinado - un enfoque que Dilthey nunca hubiese aceptado. Podemos concluir que Max Weber antepone Verstehen a Erklären, teorizando, no obstante, una propuesta atenuada del dualismo metodológico. A continuación, expongo un nuevo argumento a favor de situar a Weber en el marco de Verstehen, que se relaciona con la dicotomía predicción-comprensión.

\section{Hacia una reconfiguración: el marco de la controversia predicción-comprensión}

W. J. González ha argumentado que existe otra dualidad que acompaña a la dupla Erklären-Verstehen: se trata de la pareja predicción-comprensión. Según González, los defensores de Erklären "frecuentemente acaban otorgando un papel importante a la predicción", mientras que "los

37. Cfr. González, W. J.: "From the Characterization of 'European Philosophy of Science' to the Case of the Philosophy of the Social Sciences" en International Studies in the Philosophy of Science 29 no. 2, 2015, p. 174.

38. Sobre estos dos aspectos véase Weber, M.: "El sentido de la neutralidad valorativa de las ciencias sociológicas y económicas" en Ensayos sobre metodología sociológica. Buenos Aires: Amorrortu, 1917/2001, pp. 233-235; y Weber, M.: "La objetividad" cit., pp. 69-70, respectivamente. 
defensores de un Verstehen estricto tienden a considerar la predicción como menos relevante para la ciencia" ${ }^{39}$. Y continúa afirmando que

generalmente, el apoyo a Erklären se asocia con la compatibilidad con la predicción. De hecho, en el campo de la "explicación" la capacidad predictiva acompaña a la tarea de aportar explicaciones, pues la regularidad de los fenómenos puede darse por supuesta, lo que permite establecer predicciones sobre eventos futuros [...] No obstante, en el caso de Verstehen, la predicción no puede suponer el primer área de comprensión sobre los asuntos humanos y sociales ${ }^{40}$.

González destaca la íntima conexión metodológica -no histórica- entre predicción y ciencias de la naturaleza, y comprensión y ciencias sociales. Esto es ya recogido por Comte, quien afirma que el espíritu positivo consiste en "ver para prever, en estudiar lo que es para deducir lo que será" ${ }^{41}$. Así, a la luz del debate Erklären-Verstehen, es posible entender en qué medida la apuesta weberiana por una sociología comprensiva cercana al lado Verstehen parece yerma predictivamente. Los TI son ante todo una herramienta metodológica cuyo objetivo es la comprensión de los fenómenos sociales, es decir, su análisis desde el punto de vista cualitativo y singular. En la medida en que la sociología comprensiva se relaciona con Verstehen, la caracterización weberiana se ajusta bien a este marco teórico.

La metodología de Max Weber parece reclamar que (a) epistemológicamente, los fenómenos sociales son de una disparidad tal que hace imposible predecir los acontecimientos venideros con la misma precisión que las ciencias de la naturaleza; y como consecuencia, que (b) metodológicamente, los pasos a seguir en el análisis de la realidad social no pueden ser los mismos que aquellos de la realidad natural. Además, la finalidad de la construcción de los TI es comprender los fenómenos pasados, no los futuros, de la forma más rigurosa disponible.

Surge así una paradoja que reclama atención. Se ha afirmado que la propuesta metodológica de Max Weber, aun siendo partidaria de Verstehen, supone un desplazamiento hacia el centro, cuyo fin es atenuar la conflictiva dicotomía Erklären-Verstehen. Respecto a la caracterización weberiana, hemos mantenido anteriormente que su capacidad predictiva es nula. Un TI es incapaz de ofrecer un enunciado predictivo. Al mismo tiem-

39. González, W. J.: "From Erklären-Verstehen to Prediction-Understanding: The Methodological Framework in Economics" en Sintonen, M., Ylikoski, P. y Miller, K. (eds.).: Realism in Action: Essays in the Philosophy of Social Sciences. Dordrecht: Kluwer, 2003, p. 37.

40. Ibídem, p. 41.

41. Comte, A.: op. cit., p. 59.

Thémata. Revista de Filosofía №58 (2018) pp.: 13-34. 
po, se ha argumentado que la macro-dupla Erklären-Verstehen comprende una de alcance menor, que es la micro-dupla predicción-comprensión.

La incongruencia se hace evidente: ¿cómo puede Weber acercar las posiciones de explicación y comprensión sin tender puentes en el eje formado por la predicción y comprensión? Parece razonable que, si la metodología de Weber establece cierto grado de convergencia entre los polos Erklären-Verstehen, debería hacer lo propio con una pareja más restringida que se explica dentro de los márgenes de la primera. Tiene que encontrarse algún sentido del término de predicción compatible, en mayor o menor grado, con la metodología de carácter típico-ideal.

\section{Una redefinición de la predicción de los tipos ideales}

Weber no atiende de forma sistemática al carácter predictivo de los TI, pero su pesimismo acerca de la determinación de los desarrollos sociales es más que notable: su escrito de 1917 termina sentenciando desairado: "ello compete al futuro"42, en referencia a la dirección de las futuribles ideas alemanas del año venidero. A pesar de su impredictabilidad, Weber considera al conjunto de ciencias sociales como disciplinas científicas. Los TI contribuyen metodológicamente a la explicación en ciencias sociales, y este hecho, en la metodología weberiana, es suficiente para demarcar su carácter científico. Siendo precisos, tal forma de explicación constituye una retrodicción. Este tipo de explicación es temporal y diametralmente opuesta a la predicción: mientras que la inferencia realizada desde las causas a los efectos futuros es una predicción, la que va desde los efectos a las causas pasadas es una retrodicción.

¿Están pues los TI constreñidos a emitir retrodicciones? Creo aquí que precisar el concepto de predicción de forma gradual puede aportarnos un nuevo enfoque. Las nociones consideradas son las de previsión, predicción, pronóstico y planificación ${ }^{43}$. Las tres primeras nociones siguen un orden decreciente en cuanto a la precisión de las predicciones, mientras que la cuarta es cognitivamente dependiente de las anteriores. A la luz de esta tipología, que desglosa la predicción como una cuestión gradual, quisiera añadir que, a mi juicio, una predicción -en el sentido genérico del término- depende funda-

42. Weber, M.: "El sentido" cit., p. 269.

43. Esta tipología aparece en diversos lugares: véase Fernández Valbuena, S.: "Predicción y economía" en González, W. J. (ed.).: Aspectos metodológicos de la investigación científica. Murcia: Publicaciones Universidad de Murcia, 1990, pp. 385-405; González, W. J.: La predicción científica. Concepciones filosófico-metodológicas desde H. Reichenbach a N. Rescher. Barcelona: Montesinos, 2010, pp. 261-263; la noción de previsión con el sentido que aquí se le otorga aparece en Rescher, N.: Predicting the future. Nueva York: State University of New York Press, 1998, pp. 128 y ss.

Thémata. Revista de Filosofía №58 (2018) pp.: 13-34. 
mentalmente de tres elementos: el conocimiento de las variables, el estatus del fenómeno analizado y el alcance temporal de la predicción.

Una previsión se establece cuando las variables en juego están bajo control en un periodo de tiempo determinado y conocido. La probabilidad de acierto en las previsiones es máxima, porque las variables se conocen, el objeto de estudio es estable y el alcance temporal no es grande. Una predicción -dentro de esta nomenclatura- se da en aquellas situaciones en que las variables que son conocidas en el presente pasan a ser desconocidas en un margen temporal establecido. Fernández Valbuena asegura que una predicción así entendida se basa en una previsión, pues partimos de un valor inicial de las variables conocido ${ }^{44}$.

La probabilidad de acierto se reduce en estos casos, pues entran en juego variables desconocidas, sin que signifique por ello una considerable pérdida de capacidad predictiva, ya que el objeto de estudio sigue siendo relativamente estable y el margen de tiempo es conocido. El último eslabón es el pronóstico. Un pronóstico es un enunciado sobre el futuro en el que se admite determinado grado de error. Su carácter predictivo es débil dado que existen variables desconocidas, el objeto de estudio puede no ser estable, y el margen temporal puede ser elevado ${ }^{45}$. Un planteamiento, por otra parte, es un cálculo teleológico de acción dependiente de nuestras previsiones, predicciones y/o pronósticos ${ }^{46}$.

Los CI forman parte de las previsiones. En las situaciones en que aparecen se nos informa de forma detallada sobre las variables en juego, lo que proporciona un control absoluto sobre las situaciones futuras. Un ejemplo de previsión en este sentido es la caída de graves en el modelo galileano. Los TI, recordemos, seleccionan las características del fenómeno que considera oportuno y desestima otras. Teniendo en cuenta que, además, su objeto de estudio son fenómenos de corte histórico, en los que las variables participantes son potencialmente innumerables, es posible afirmar con rotundidad que los TI fracasan en este aspecto. En la medida en que los CI satisfacen la noción más intensa de predicción, la previsión, obviaré el resto. Mi interés es considerar si los TI son capaces de satisfacer alguna noción de predicción menos restrictiva.

Las ciencias de la naturaleza frecuentemente presentan previsiones de carácter fuerte, mientras que las ciencias sociales son más débiles

44. Cfr. Fernández Valbuena, S.: op. cit., pp. 388-389.

45. González admite que este tipo de predicción puede considerarse como cualitativa, frente a las predicciones y previsiones, que constituyen predicciones cuantitativas. Esto es importante para las ciencias sociales, donde muchas veces importa más la tendencia esperable que los detalles concretos de la tendencia. Véase González, W. J.: La predicción científica cit., p. 262. 46. Cfr. Ibídem, p. 262.

Thémata. Revista de Filosofía ํ58 (2018) pp.: 13-34. 
en este aspecto. El asunto central es cómo unas y otras manejan las infinitas variables que afectan a la explicación, sea esta o no predictiva. Las primeras hacen uso de los CI, modificando deliberadamente las variables oportunas de su objeto de estudio -generalmente, otorgándoles un valor igual a 0. Por contraposición, los TI recortan las variables del fenómeno analizado, abandonando muchas de ellas sin consideración. Consecuentemente, a la vista de la amalgama de variables desatendidas, predecir el estado futuro de dichos fenómenos es inviable.

Pero, al mismo tiempo, el proceso abstractivo no es aleatorio: el científico social recoge las características del objeto de estudio que considera más relevantes. ¿No debería ser esto indicativo del posible devenir del fenómeno? En rigor, un fenómeno de corte social es comprendido a partir de las relaciones que se establecen entre conjuntos de TI. Por ello, en caso de identificar sistemas de relaciones semejantes, en un tiempo presente, entre aquellos TI -o aproximados- que explicaron un fenómeno determinado en el pasado, ¿no estaríamos autorizados a pronosticar una futura tendencia similar? Por descontado que, sin un dominio total de las variables y propiedades de aquello estudiado, las previsiones se antojan inconcebibles. Pero si, de acuerdo con Max Weber, un TI selecciona, de todas las variables, las que objetivamente describen el fenómeno con mayor precisión, establecer pronósticos a partir de ellos puede resultar eficaz. Quizá, desde esta perspectiva, un TI pueda otorgar información justificada sobre posibles tendencias.

Volvamos a la tesis sostenida por Weber en La ética protestante... La ética calvinista proporcionó un sustrato óptimo a la expansión económica capitalista de un grupo de países norte-europeos. Con "ética calvinista" y "capitalismo", en tanto TI, Weber describe una suerte de fenómenos que incluyen enunciados del tipo "deificación del trabajo y la vida laboriosa", "acumulación de bienes y dinero" o "condena del despilfarro". Estas actitudes son variables o propiedades de los TI, cuya conexión causal ofrece un modelo comprensivo sobre el fenómeno descrito.

Imaginemos ahora que, en un conjunto de países subdesarrollados actuales, comienzan a proliferar creencias religiosas semejantes a las calvinistas y se produce una inesperada y progresiva acumulación de capital. Afirmar que los TI weberianos nos permiten predecir el futuro de dichos países es demasiado, pues ni a) dominamos todas las variables, ni b) el fenómeno analizado es estable, ni c) el alcance temporal de la predicción es lo suficientemente corto. Aun así, mantengo que sí es legítimo formular un pronóstico tal y como ha sido definido a partir de la metodología típico-ideal. La experiencia sobre fenómenos pasados puede indicarnos las tendencias a seguir de fenómenos presentes que muestran variables parecidas a las recogidas por los TI pasados. Lo que se defiende con todo

Thémata. Revista de Filosofía Nº5 (2018) pp.: 13-34. 
esto es que la capacidad predictiva de los TI es, cuando menos, limitada. Ahora bien: desglosando la noción de predicción y diferenciando grados de precisión según el manejo de variables, el objeto de estudio, y el desfase temporal con que se establece la predicción, parece ciertamente apropiado hablar de la capacidad de los TI de establecer pronósticos.

\section{Referencias}

Camardi, G.: "Ideal Types and Scientific Theories" en F. Coniglione et. al. (eds.). Idealization XI: Historical Studies on Abstraction and Idealization. Ámsterdam: Rodopi, 2004, pp. 273-285.

Cartwright, N.: Nature's Capacities and their Measurement. Oxford: Oxford, 1989.

Comte, A.: Discurso sobre el espíritu positivo. Buenos Aires: Aguilar, 1844/1982.

De Donato, X.: "El carácter de los tipos ideales weberianos y su relación con las ciencias naturales" en Diánoia 52 no. 59, 2007, pp. 151-177.

Dilthey, W.: Introducción a las ciencias del espíritu, en las que se trata de fundamentar el estudio de la sociedad y de la historia. México: Fondo de cultura económica, 1883/1949.

Drysdale, J.: "How are Social-Scientific Concepts Formed? A Reconstruction of Max Weber's Theory of Concept Formation" en Sociological Theory 14 no. 1, 1996, pp. 71-88.

Fernández Valbuena, S.: "Predicción y economía" en González, W. J. (ed.).: Aspectos metodológicos de la investigación científica. Murcia: Publicaciones Universidad de Murcia, 1990, pp. 385-405.

Freund, J.: Sociología de Max Weber. Barcelona: Ediciones Península, 1966/1986.

González, W. J.: "From Erklären-Verstehen to PredictionUnderstanding: The Methodological Framework in Economics" en Sintonen, M., Ylikoski, P. y Miller, K. (eds.).: Realism in Action: Essays in the Philosophy of Social Sciences. Dordrecht: Kluwer, 2003, pp. 33-50.

González, W. J.: La predicción científica. Concepciones filosófico-metodológicas desde H. Reichenbach a N. Rescher. Barcelona: Montesinos, 2010.

González, W. J.: Philosophico-Methodological Analysis of Prediction and its Role in Economics. Dordrecht: Springer, 2015.

González, W. J.: "From the Characterization of "European Philosophy of Science' to the Case of the Philosophy of the Social Sciences" en International Studies in the Philosophy of Science 29 no. 2, 2015, pp. 167-188.

Hempel, C. G. La explicación científica. Estudios sobre la Filosofía de la ciencia. Buenos Aires: Paidós, 1965/1978. 
Ibarra, A. y Mormann, T.: "Datos, fenómenos y constructos teóricos: un enfoque representacional" en Theoria 13 no. 1, 1998, pp. 61-87.

Nagel, E.: "Assumptions in Economic Theory" en The American Economic Review 53, no. 2, 1963, pp. 211-219.

Nowak, L.: The structure of idealization. Towards a systematic interpretation of the marxian idea of science. Dordrecht: Springer, 1980.

Nowak, L.: "The idealizational approach to science: a new survey" en Brezinski, J. y Nowak, L. (eds.). Idealization III: Approximation and Truth. Poznan Studies in the Philosophy of the Sciences and the Humanities. Ámsterdam/Atlanta: Rodopi, 1992, pp. 9-63.

Papineau, D.: "Ideal Types and Empirical Theories" en The British Journal for the Philosophy of Science 27 no. 2, 1976, pp. 137-146.

Kuhn, T. S.: "Epílogo: 1969" en La estructura de las revoluciones científicas. Madrid: Fondo de Cultura Económica, 1962/2007, pp. 301-353.

Kuhn, T. S.: Segundos pensamientos sobre paradigmas. Madrid: Tecnos, 1974/1978.

Liu, C.: "Approximation, Idealization, and Laws of Nature" en Synthese 118, 1999, pp. 229-256.

Ramsey, J. L.: "Ideal Reaction Types and the Reactions of Real Alloys" en Proceedings of the Biennial Meeting of the Philosophy of Science Association 1, 1994, pp. 149-159.

Rescher, N.: Predicting the future. Nueva York: State University of New York Press, 1998.

Sánchez de Puerta, F. J.: "Los tipos ideales en la práctica: significado, construcciones, aplicaciones" en Empiria. Revista de Metodología de Ciencias Sociales 11, 2006, pp. 11-32.

Stegmüller, W.: Teoría y experiencia. Barcelona: Ariel, 1970/1979.

Watkins, J. W. N.: "Ideal Types and Historical Explanation" en The British Journal for the Philosophy of Science 3 no. 9, 1952, pp. 22-43.

Weber, M.: "La objetividad cognoscitiva de la ciencia social y de la política social" en Ensayos sobre metodología sociológica. Buenos Aires: Amorrortu, 1904/2001, pp. 39-101.

Weber, M.: La ética protestante y el espíritu del capitalismo. Barcelona: Península, 1905/1969.

Weber, M.: "Sobre algunas categorías de la sociología comprensiva" Ensayos sobre metodología sociológica. Buenos Aires: Amorrortu, 1913/2001, pp. 175-221.

Weber, M.: "El sentido de la neutralidad valorativa de las ciencias sociológicas y económicas" en Ensayos sobre metodología sociológica. Buenos Aires: Amorrortu, 1917/2001, pp. 222-269. 
